\title{
Development and application of a method to detect 27 respiratory pathogens using multiplex RT-PCR combined with MassARRAY technology
}

Huan Zhao ${ }^{1}$, Yichao Yang ${ }^{2}$, Jiangfeng Lyu ${ }^{2}$, Xuyi Ren ${ }^{2^{*}}$ and Wei Cheng ${ }^{3^{*}}$

\begin{abstract}
Background: Respiratory tract infections are the most common infections that lead to morbidity and mortality worldwide. Early recognition and precise diagnosis of microbial etiology is important to treat LRTIs promptly, specifically and effectively.
\end{abstract}

Objectives: To establish a method based on multiplex reverse transcription (MRT)-PCR and MasSARRAY technology for the simultaneous detection of 27 respiratory pathogens and explore its clinical application value.

Methods: Analytical sensitivity and specificity of the MRT-PCR-MasSARRAY system were validated using inactivated bacterial and viral strains. Also we analyzed samples from 207 patients by MassARRAY methods and compared the results with consensus $\mathrm{PCR} /$ reverse transcription (RT)-PCR.

Results: The minimum detection limit of our MRT-PCR-MassARRAY method for pathogens was 10-100 copies/ $\mu$ l, with high specificity. Comparison test with consensus PCR/RT-PCR on 207 clinical samples, the positive, negative, and total correlation rates were 100,98.68, and 99.03\%, respectively. There was a high degree of agreement between the test results of the two methods ( $P<0.01$ by McNemar's test).

Conclusion: Our detection system of 27 respiratory pathogens based on MassARRAY technology has high sensitivity and specificity, high throughput, and is simple to operate. It provides diagnostic value for the clinical diagnosis of respiratory pathogens and is of great significance in the screening of respiratory pathogens.

Keywords: Respiratory pathogens, Multiplex RT-PCR, MassARRAY technology

\footnotetext{
*Correspondence: destinylv@163.com; 153052140@qq.com

${ }^{2}$ Research and Development Centre, Hangzhou DiAn Medical Laboratory, Hangzhou, Zhejiang, China

${ }^{3}$ Department of Respiratory Diseases, Nantong Tongzhou People's Hospital, Nantong, Jiangsu, China

Full list of author information is available at the end of the article
}

(c) The Author(s). 2021 Open Access This article is licensed under a Creative Commons Attribution 4.0 International License, which permits use, sharing, adaptation, distribution and reproduction in any medium or format, as long as you give appropriate credit to the original author(s) and the source, provide a link to the Creative Commons licence, and indicate if changes were made. The images or other third party material in this article are included in the article's Creative Commons licence, unless indicated otherwise in a credit line to the material. If material is not included in the article's Creative Commons licence and your intended use is not permitted by statutory regulation or exceeds the permitted use, you will need to obtain permission directly from the copyright holder. To view a copy of this licence, visit http://creativecommons.org/licenses/by/4.0/ The Creative Commons Public Domain Dedication waiver (http://creativecommons.org/publicdomain/zero/1.0/) applies to the data made available in this article, unless otherwise stated in a credit line to the data. 


\section{Background}

Respiratory tract infections are common infectious diseases in clinics. They are highly prevalent in infants, the elderly and immunocompromised patients, often leading to severe symptoms or mortality [1]. According to the WHO, approximately 3 million people died from lower respiratory tract infections in 2016 [2]. Common pathogens of respiratory tract infections include viruses, bacteria, mycoplasmas, chlamydiae, and other microorganisms [3]. Viral pathogens mainly include influenza A virus, influenza B virus, parainfluenza virus (PIV), respiratory syncytial virus, rhinovirus, human metapneumovirus (HMPV), and adenovirus [4]. Bacterial pathogens include Streptococcus pneumoniae, Haemophilus influenzae, and Moraxella catarrhalis [5]. Most respiratory tract infections have similar clinical symptoms, making their identification difficult for clinicians. Thus, the rapid identification of pathogens of respiratory tract infections is of great significance for accurate clinical diagnosis and the administration of appropriate medication. Over the past few years, the rapid development of molecular detection technology has largely compensated for the shortcomings of traditional culture and immunoassay methodologies used to detect respiratory pathogens, substantially improving the speed, sensitivity, and specificity of the detection and identification of respiratory pathogens. In particular, the development of pathogen detection systems based on multiplex PCR allows the simultaneous detection of many different pathogens for use in clinical applications [6-9]. Multiplex real-time quantitative fluorescence PCR detection has relatively high sensitivity and specificity but is limited by the number of fluorescence types in each reaction. Generally, only 3-4 pathogens can be detected in one reaction, and if more pathogens need to be detected, more reactions are required, substantially reducing the convenience of detection and throughput. Luminex's NxTAG Respiratory Viral Panel is based on liquid chip technology and can simultaneously detect 20 pathogens, including 18 viruses and subtypes, as well as Mycoplasma pneumoniae and Chlamydia pneumoniae [10]. However, this commercial kit has not been widely used in clinics due to the high cost of its reagents.

Agena Bioscience's MassARRAY System integrates the sensitivity of PCR technology and the high throughput of mass spectrometry detection technology. One reaction can achieve up to 40 gene amplifications that can be applied to single nucleotide polymorphism analysis, mutant detection, DNA methylation analysis, gene copy number identification, and other research areas [11]. Currently, the main applications of MassARRAY in clinics are deafness gene detection, epidermal growth factor receptor mutation detection, liquid biopsy, and hypertension gene detection [12-15]. In this study, we developed a new method based on multiplex reverse transcriptase (MRT)-PCR combined with MassARRAY technology to detect 27 common pathogens causing respiratory tract infections. We also evaluated its application value in clinics in the diagnosis of pathogens of respiratory tract infections.

\section{Methods \\ Sample collection}

We collected nasopharyngeal aspirate samples from 207 patients with suspected respiratory tract infection who were submitted to the sixth people's hospital of Nantong from September 2019 to March 2020. The patients were aged 1-76 years only and comprised 112 males and 95 females. The collection of all NPA samples was approved by the ethics committee of the sixth people's hospital of Nantong. And informed consent was obtained from all subjects. We confirmed that all methods in this study were carried out in accordance with relevant guidelines and regulations.

\section{Primers used for multiplex PCR and extension reaction}

Specific amplification primers and extension primers for all targets including several influenza A virus (Flu-A $\mathrm{H} 1 \mathrm{~N} 1, \mathrm{H} 3, \mathrm{H} 5, \mathrm{H} 7, \mathrm{H} 3 \mathrm{~N} 2$ seasonal type), influenza B virus (Flu-B), parainfluenza virus types 1 to 4 (PIV1 to 4), four human coronaviruses (HCoV-OC43, 229E, NL63, and HKU1), respiratory syncytial viruses (RSV), human metapneumoviruses (HMPV), human bocavirus (HBoV), human rhinovirus (HRV), human enterovirus (HEV), adenovirus (ADV), Moraxella catarrhalis (M. catarrhalis), Mycoplasma pneumoniae (MP), Chlamydia pneumonia (CP), Bordetella pertussis (B.pertussis), Haemophilus influenza(H.influenza), Streptococcus pneumoniae(S. pneumoniae), Streptococcus pyogenes (S.pyogenes), Streptococcus agalactiae (S.agalactiae) and Legionella pneumophila (L.pneumophila) were designed by MassARRAY Assay Design software using published sequences from Gene Bank (Table 1). All primers were synthesized by Invitrogen Corp (Shanghai, China).

\section{Nucleic acids extraction}

Extraction of nucleic acids (NA) from $200 \mu \mathrm{l}$ of NPA was performed by MagNA Pure LC extraction using the total nucleic acid extraction kit (Roche Diagnostics, Penzberg, Germany), and the operation steps were performed in strict accordance with the instructions of the kit. Sample quality control was evaluated by detection of the glyceraldehyde 3-phosphate dehydrogenase (GAPDH) gene as an internal control.

\section{Multiplex RT-PCR and MassARRAY detection}

First, we performed MRT-PCR using a $40 \mu \mathrm{l}$ reaction mix contained $4 \mu \mathrm{l} 10 \times$ PCR buffer (Takara, Japan), 0.25 $\mathrm{mM}$ dNTP (Takara), $4 \mathrm{mM} \mathrm{MgCl} 2,0.5 \mu \mathrm{M}$ of each amplification primer, $20 \mathrm{u}$ PrimeScript ${ }^{\mathrm{TM}}$ II reverse 
Table 1 Sequences of amplification primers for 27 pathogens

\begin{tabular}{|c|c|c|}
\hline Pathogen & Forward Primer $(5 \rightarrow 3)$ & Reverse primer $(5 \rightarrow 3)$ \\
\hline M.catarrhalis & ACGTTGGATGTAGCAGGCGGTGTTGGTGTT & ACGTTGGATGTTGTAGCTTGCTTCAACGCC \\
\hline HRV & ACGTTGGATGAACAGTGGTCCAGCCTGCGT & ACGTTGGATGAACACGGACACCCAAAGTAG \\
\hline MP & ACGTTGGATGCCCGAACAAAATAATGATTCC & ACGTTGGATGGTTTGACAAAGTCCGTGAAG \\
\hline $\mathrm{HCOV}$ & ACGTTGGATGGGGAGTAATGAACCCGGTAA & ACGTTGGATGGAGCTAATAACACGGCTCTG \\
\hline Flu-A & ACGTTGGATGAAGACCAATCCTGTCACCTC & ACGTTGGATGAAAGCGTCTACGCTGCAGTC \\
\hline $\mathrm{CP}$ & ACGTTGGATGCGGAAGGGTTAGTAGTACAT & ACGTTGGATGCGCATAAACTCTTCCTCAAC \\
\hline Flu-B & ACGTTGGATGACGGTGGATTAAACAAAAGC & ACGTTGGATGGCAATAGCTCCGAAGAAACC \\
\hline HMPV & ACGTTGGATGAGCTTCAGTCAGTTCAACAG & ACGTTGGATGCCTGCAGATGTTGGCATGTA \\
\hline B. pertussis & ACGTTGGATGTGGGAGTTCTGGTAGGTGTG & ACGTTGGATGATCAAGCACCGCTTTACCC \\
\hline H. influenzae & ACGTTGGATGTTGGCCCAGGTTGGTATATG & ACGTTGGATGTTACGCACGGTAAGGATG \\
\hline H5 & ACGTTGGATGTGACTATCCACAATACTCAG & ACGTTGGATGGACCAGCTATCATGATTGCC \\
\hline S.pyogenes & ACGTTGGATGAGCAACAAGTAGTACAGCAG & ACGTTGGATGTTAGCTACTAGTGTAGCTG \\
\hline S.pneumoniae & ACGTTGGATGTCGTGCGTTTTAATTCCAGC & ACGTTGGATGACGCAATCTAGCAGATGAAG \\
\hline PIV4 & ACGTTGGATGGATCCACAGCAAAGATTCAC & ACGTTGGATGCCTGCAAGGAAAGCAGAGAT \\
\hline H1N1 & ACGTTGGATGGTTAACATCAGCAACACCAAC & ACGTTGGATGAGAGAGGAATTGCCCGC \\
\hline S.agalactiae & ACGTTGGATGGAAACATTGATTGCCCAGC & ACGTTGGATGAGGAAGATTTATCGCACCTG \\
\hline PIV3 & ACGTTGGATGACTCTATCCACTCTCAGACC & ACGTTGGATGATGGGATCTGAGGATAC \\
\hline PIV2 & ACGTTGGATGCTGCAGCTATGAGTAATCAC & ACGTTGGATGGATCGAGCATCTGGAATAAC \\
\hline ADV & ACGTTGGATGGACGCCTCGGAGTACCTGA & ACGTTGGATGAGCCACCGTGGGGTTTCTAA \\
\hline RSV & ACGTTGGATGCACAGAAGATGCTAATCAT & ACGTTGGATGGGTCTTCTCTTCCTAATC \\
\hline H3 & ACGTTGGATGCAGTACAGGGAATCTAATTG & ACGTTGGATGGCATTCAGAATTGCATTTG \\
\hline L.pneumophila & ACGTTGGATGTGGTGACTGCAGCTGTTATG & ACGTTGGATGTCCGGATTAACATCTATGCC \\
\hline PIV1 & ACGTTGGATGATTACCTGGACCAAGTCTAC & ACGTTGGATGCACATCCTTGAGTGATTAAG \\
\hline HEV & ACGTTGGATGCTGAATGCGGCTAATCCCAA & ACGTTGGATGGATTGTCACCATAAGCAGCC \\
\hline $\mathrm{H} 7$ & ACGTTGGATGCAGCATACAATTGATCTGGC & ACGTTGGATGCCATCTTCTTCAGCATTCTC \\
\hline H3N2 seasonal & ACGTTGGATGACCACCCGGGTACGGACAA & ACGTTGGATGGGCTTCTTTTGGTAGATACTG \\
\hline HBoV & ACGTTGGATGAAATCTCTTCTGGCTACACG & ACGTTGGATGCCTCTGCGATCTCTATATTG \\
\hline GAPDH & ACGTTGGATG CTGCTCACATATTCTGGAGGAG & ACGTTGGATG AAAAGCAGCCCTGGTGACC \\
\hline
\end{tabular}

transcriptase (Takara), 1u TaKaRa Hot start-Taq polymerase. The MRT-PCR was carried out on AB 2720 PCR instrument ( $A B$, USA) for thermocycling and the cycling conditions were $50^{\circ} \mathrm{C} 25 \mathrm{~min}, 95^{\circ} \mathrm{C} 5 \mathrm{~min}$, followed by 45 cycles of $\left(94^{\circ} \mathrm{C} 15 \mathrm{~s}, 55^{\circ} \mathrm{C} 30 \mathrm{~s}, 68^{\circ} \mathrm{C} 20\right.$ s). Second, we used shrimp alkaline phosphatase to remove dNTPs from the PCR product (dephosphorylation), $5 \mu \mathrm{l}$ RT-PCR product from the first step was taken off and mixed with $2 \mu$ shrimp alkaline phosphatase (Agena, USA), incubated at $45^{\circ} \mathrm{C}$ for $25 \mathrm{~min}, 80^{\circ} \mathrm{C}$ for 2 min. Third, we added $2 \mu \mathrm{l}$ of single-base extension solution (Agena Bioscience) and $0.5 \mu \mathrm{M}$ of each extention primer to the product of the second step, and then performed single-base extension as follows: $94^{\circ} \mathrm{C}$ for $30 \mathrm{~s}$, followed by 30 cycles of $\left(94^{\circ} \mathrm{C}\right.$ for $5 \mathrm{~s}$, five cycles of $\left[52^{\circ} \mathrm{C}\right.$ for $5 \mathrm{~s}, 80^{\circ} \mathrm{C}$ for $\left.5 \mathrm{~s}\right]$ ), and $72^{\circ} \mathrm{C}$ for $3 \mathrm{~min}$. After resin desalination, $10 \mu \mathrm{l}$ of the extended PCR product was loaded onto the MassARRAY chip (Agena
Bioscience), and the mass spectrometry results were analyzed with Typer 4.0 software (Agena Bioscience).

\section{Sensitivity and specificity assessment of MRT-PCR- MassARRAY}

The sensitivity of the MRT-PCR-MassARRAY for pathogen detection was studied using inactivated bacterial and viral strains with a quantitative certification, which were purchased from National Institutes for Food and Drug Control, China. The strains underwent serial dilution to obtain 10,000, 1000, 100, and 10 copies/ $\mu \mathrm{l}$. The detection results were automatically interpreted by the instrument. A positive result was indicated by a reduced or totally consumed extension primer peak and the extension product peak amplifying one base at the corresponding position. The extension primer peak sequence, molecular mass of each pathogen, and the extension product sequence and molecular mass are shown in 
Table 2. Each diluted pathogen was tested in triplicate, and the lowest dilution concentration that was positive in all three replicates was defined as the limit of detection. We investigated the specificity of the detection system by performing MRT-PCR on 10,000 copies/ $\mu$ l of each pathogen. If each pathogen extension product peak occurred at a specific location and there was no cross-overlap between each pathogen extension product peak, the specificity of the detection system was considered to be good.

\section{Clinical application of respiratory pathogen detection (MRT-PCR-MassARRAY)}

The collected nasopharyngeal swab samples were tested with MRT-PCR-MassARRAY, as described above. All target respiratory viruses were screened using consensus $\mathrm{PCR} /$ reverse transcription (RT)-PCR assays, according to previous reports [16-25]. PCR was performed using the
FastStart high-fidelity PCR system (Roche Molecular Systems, Inc., Pleasanton, CA, USA). RT-PCR was performed using the One-Step RT-PCR kit (Invitrogen). Amplified DNA was purified using a QIAquick gel extraction kit, according to the manufacturer's protocol (Qiagen, Valencia, CA, USA) and sequenced on an ABI3730 automated sequencer (Applied Biosciences, Foster City, CA, USA). Differences between the detection rates of the two methods were tested using the McNemar's test. A $P$ value of $<0.01$ was considered statistically significant.

\section{Results}

Sensitivity and specificity of MRT-PCR-MassARRAY for respiratory pathogen

Detection

For the negative control and pathogens below the detection limit, only the extension primer peak was

Table 2 Sequence and molecular mass of extension primer and extension product

\begin{tabular}{|c|c|c|c|c|c|}
\hline Pathogen species & $\begin{array}{l}\text { Extension Primer } \\
\text { Sequence }\end{array}$ & $\begin{array}{l}\text { Molecular mass of } \\
\text { extension primer }\end{array}$ & $\begin{array}{l}\text { Extended } \\
\text { base }\end{array}$ & $\begin{array}{l}\text { Extension Product } \\
\text { Sequence }\end{array}$ & $\begin{array}{l}\text { Elongation Product } \\
\text { Molecular Mass }\end{array}$ \\
\hline M.catarrhalis & TCAACGCCCACATTT & 4471.9 & G & TCAACGCCCACATTTG & 4759.1 \\
\hline HRV & CTTTGAGTCCTCCGGC & 4824.1 & C & CTITGAGTCCTCCGGCC & 5071.3 \\
\hline MP & AAAGCCACCCTGATCAC & 5108.4 & C & AAAGCCACCCTGATCACC & 5355.5 \\
\hline $\mathrm{HCOV}$ & ACGGCTGTGTAAAGA & 5234.4 & $\mathrm{~T}$ & ACGGCTGTGTAAAGAT & 5561.5 \\
\hline Flu-A & CACGCTGCAGTCCTCGCT & 5411.5 & C & CACGCTGCAGTCCTCGCTC & 5658.7 \\
\hline$C P$ & TTCCTCAACCGAAAGGTC & 5443.6 & C & TTCCTCAACCGAAAGGTCC & 5690.7 \\
\hline Flu-B & AAAGGCCATAGGGAATTG & 5596.7 & C & AAAGGCCATAGGGAATTGC & 5843.9 \\
\hline HMPV & ACGCGGCAGTTTCAGACA & 5812.8 & A & ACGCGGCAGTITCAGACAA & 6084 \\
\hline B. pertussis & CTTCCCGCCCAGACCAAT & 5966.9 & G & CTTCCCGCCCAGACCAATG & 6254.1 \\
\hline H. influenzae & GATGCACTTCCACATTATAT & 6051 & $\mathrm{~T}$ & GATGCACTTCCACATTATT & 6378.1 \\
\hline H5 & GATAAACAGTGGCGAGTTCC & 6166 & C & GATAAACAGTGGCGAGTTCCC & 6413.2 \\
\hline S.pyogenes & CCCCCTCCAGGAGCAACTTGA & 6336.1 & G & CCCCCTCCAGGAGCAACTTGAG & 6623.3 \\
\hline S.pneumoniae & GTATCAGATGAAGCAGGTTTG & 6525.3 & C & GTATCAGATGAAGCAGGTTTGC & 6772.4 \\
\hline PIV4 & GGTAGTAATGCCCCTTGCTTAA & 6725.4 & $\mathrm{~T}$ & GGTAGTAATGCCCCTTGCTTAAT & 7052.5 \\
\hline H1N1 & CTGTACAGTCAGTGGTTTCCGT & 6732.4 & G & CTGTACAGTCAGTGGTTCCGTG & 7019.6 \\
\hline S.agalactiae & CCAGGCCCCCCACGATACTCATG & 6914.5 & C & CCAGGCCCCCCACGATACTCATGC & 7161.7 \\
\hline PIV3 & GATCTCTGAGGATACAGATGAAT & 7111.7 & G & GATCTCTGAGGATACAGATGAATG & 7398.9 \\
\hline PIV2 & СССTGTTATTTCTACTCTATCTAT & 7194.7 & G & СССTGTTATTTCTACTCTATCTATG & 7481.9 \\
\hline ADV & CCCCCAAGTACGTGTCGGTGGCAC & 7314.7 & G & CCCCCAAGTACGTGTCGGTGGCACG & 7602 \\
\hline RSV & СТАTTСТСТTCСТAATCTAGACATA & 7525.9 & G & СTATTCTCTTCCTAATCTAGACATAG & 7813.1 \\
\hline $\mathrm{H} 3$ & CGCTAAATAATGAGATCAGATGCAC & 7683 & C & CGCTAAATAATGAGATCAGATGCACC & 7930.2 \\
\hline L.pneumophila & GTATIITTAAAATTCTTCCCCAAATC & 7854.1 & C & GTATIITAAAATTCTTCCCCAAATCG & 8141.3 \\
\hline PIV1 & GGGACGAATACGCATATTGCATCA & 8003.2 & G & GGGACGAATACGCATATTGCATCAC & 8250.4 \\
\hline HEV & AAAGGAAACACGGACA & 4941.3 & G & AAAGGAAACACGGACAC & 5188.4 \\
\hline H7 & ATGGACAAACTGTACGA & 5227.4 & A & ATGGACAAACTGTACGAA & 5498.6 \\
\hline H3N2 seasonal & AATCTTCCTGTATGCTCA & 5424.5 & A & AATCTTCCTGTATGCTCAA & 5695.8 \\
\hline HBoV & CTCTATATTGAAGGATCTGCAT & 6724.4 & C & CTCTATATTGAAGGATCTGCATG & 7011.6 \\
\hline GAPDH & ATACGACCAAATCTAAGAGAC & 6416.3 & G & ATACGACCAAATCTAAGAGACG & 6769.6 \\
\hline
\end{tabular}


detected but no extension product peak, while at dilutions above the detection limit, the extension primer is consumed, and only the specific extension product peak can be seen (Fig. 1). The analysis of the detected peaks of 27 pathogens showed only a single extension product for each pathogen, indicating that the specificity of the MRT-PCRMassARRAY detection system was good. And the

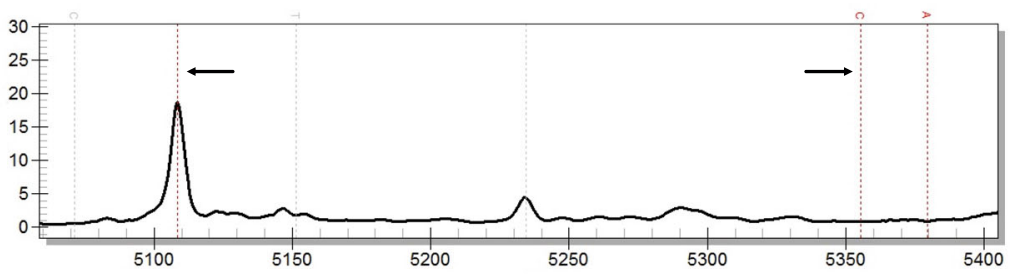

(A)

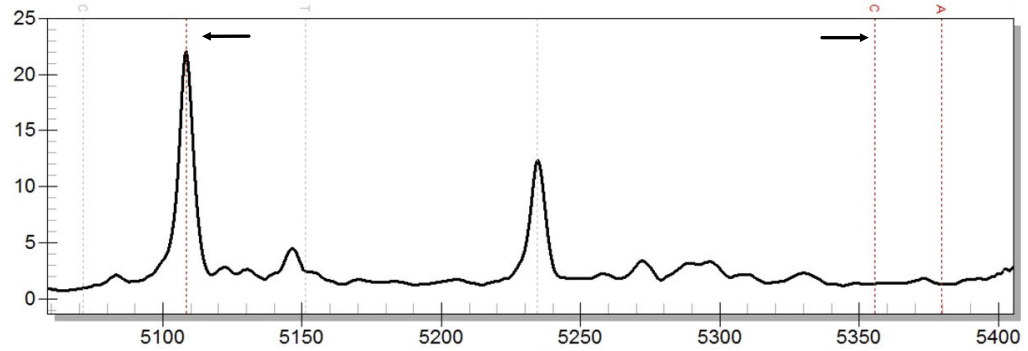

(B)

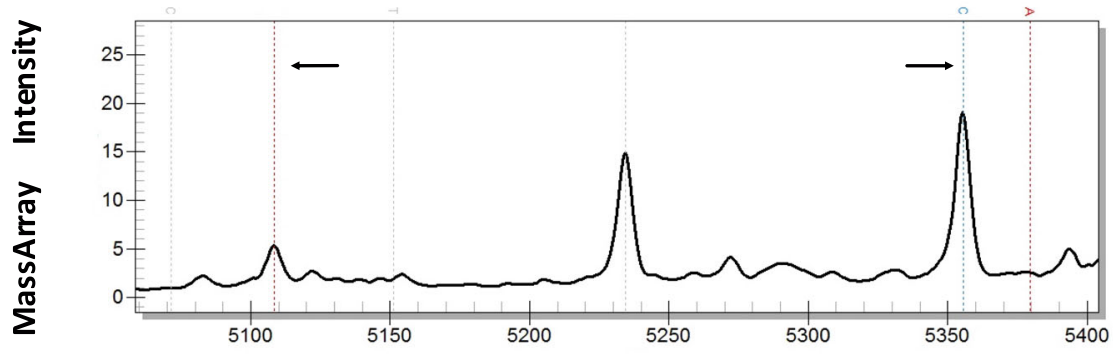

(C)

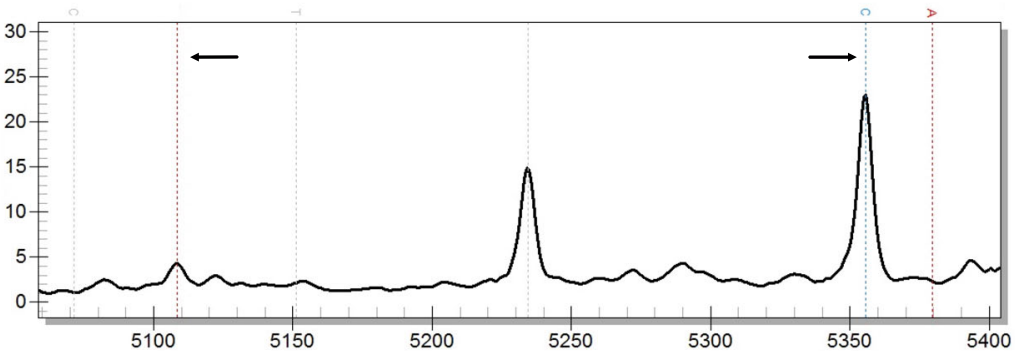

(D)

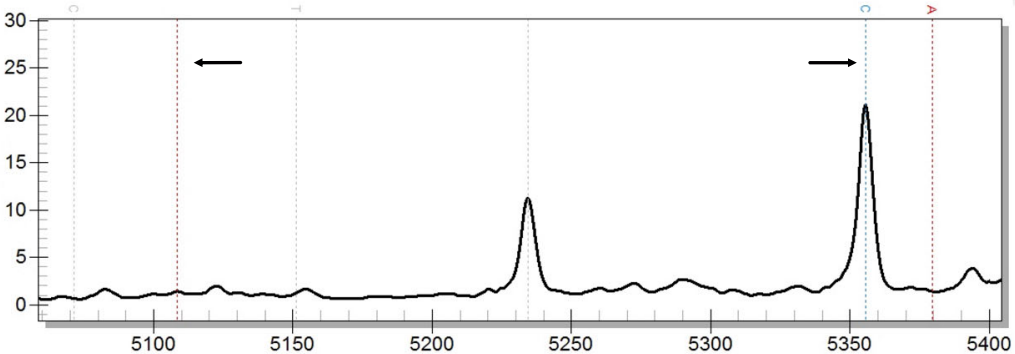

(E)

Fig. 1 Mass spectrum results showing the sensitivity of the detection of Mycoplasma pneumoniae. A negative control, B-E M pneumoniae strain diluted to 10,100,1000, and 10,000 copies/ $\mu$, respectively. The arrow on the left side of the spectrum results indicates the position of the extension primer peak, the arrow on the right side indicates the position of the extension product, and the peak at approximate 5235 Da is the non-extended primer of $\mathrm{HCoV}$. When concentrations of the negative control and pathogen were $10 \mathrm{copies} / \mu \mathrm{l}$, there was only an extension primer peak and no extension product peak. At plasmid concentrations of 100, 1000, and 10,000 copies/ $\mu$, extension product peaks appeared, and the extension primer peaks became smaller or absent due to consumption. Thus, the detection sensitivity for $M$. pneumoniae was 100 copies $/ \mu l$ 
Table 3 Detection limit of the MRT-PCR-MasSARRAY

\begin{tabular}{llll}
\hline Pathogens & LOD (copies/ul) & Pathogens & LOD (copies/ul) \\
\hline M.catarrhalis & 100 & H1N1 & 100 \\
HRV & 100 & S.agalactiae & 10 \\
MP & 100 & PIV3 & 100 \\
HCoV & 100 & PIV2 & 100 \\
Flu-A & 100 & ADV & 100 \\
CP & 10 & RSV & 1000 \\
Flu-B & 1000 & H-3 & 100 \\
HMPV & 100 & L.pneumophila & 100 \\
B.pertussis & 100 & PIV1 & 10 \\
H. influenzae & 100 & HEV & 10 \\
H-5 & 10 & H-7 & 10 \\
S.pyogenes & 100 & H3N2 seasonal & 100 \\
S.pneumoniae & 100 & HBoV & 10 \\
PIV4 & 10 & & \\
\hline
\end{tabular}

detection limit of all pathogens was between 10 copies/ $\mu$ l to 100 copies/ $\mu$ l (Table 3).

\section{Clinical performance of the MRT-PCR-MassARRAY detection system}

Of the 207 clinical samples that were detected and analyzed using the MRT-PCR-MassARRAY detection system, $57(27.54 \%)$ cases were positive, of which 33 cases were infected with a single pathogen, 19 cases with two pathogens, and five cases with three to four pathogens. S. pneumoniae was detected in 20 cases (9.66\%), M. catarrhalis in $12(5.80 \%)$, M. pneumoniae in 11
(5.31\%), and $H$. influenzae in 1 (5.31\%), PIV in ten (4.83\%; including types $1-4$ types), HMPV in seven (3.38\%), and influenza virus type A in 6 (2.90\%). The details of other detected pathogens are presented in Table 4.

\section{Comparison of MRT-PCR MassARRAY and the consensus PCR/RT-PCR method}

There were only two sample discrepancies in the test results between the two assays, both of which were positive by MassARRAY but negative by consensus PCR/ RT-PCR. The positive, negative, and total correlation rates were 100, 98.68, and 99.03\%, respectively. There was a high degree of agreement between the test results of the two methods $(P<0.01$ by McNemar's test).

\section{Discussion}

The global incidence of respiratory tract infections is high, leading to millions of deaths annually. In one hospital, $36.7 \%$ of deaths in patients $<12$ years old were due to pneumonia caused by respiratory tract infections [24]. The wide variety of pathogens and similar clinical symptoms of respiratory tract infections pose great difficulties to clinicians for diagnosis and treatment. In this study, we had developed a new method using multiplex RTPCR combined with mass spectrometry to detect common pathogens infected in respiratory tract, and also we had evaluated its analytical and clinical performace.

Of the 27 pathogens in our study, only influenza B virus and respiratory syncytial virus had a detection sensitivity of $1000 \mathrm{copies} / \mu \mathrm{l}$, and the remainder was

Table 4 Detection of Each Pathogen

\begin{tabular}{lllll}
\hline Pathogen & Single infection $(\boldsymbol{n}=\mathbf{3 3})$ & Dual infections $(\boldsymbol{n}=\mathbf{1 9})$ & $\begin{array}{l}\text { Multiple infections } \\
(\boldsymbol{n}=\mathbf{5})\end{array}$ & 0 \\
ADV & 0 & $1(0.48 \%)$ & $1(0.48 \%)$ & $1(0.48 \%)$ \\
BP & $3(1.45 \%)$ & $3(1.45 \%)$ & $1(0.48 \%)$ & $7(3.38 \%)$ \\
FluA & $2(0.97 \%)$ & $3(1.45 \%)$ & $2(0.97 \%)$ & $6(2.90 \%)$ \\
Hin & $4(1.93 \%)$ & $5(2.41 \%)$ & 0 & $11(5.31 \%)$ \\
HCOV & 0 & $1(0.48 \%)$ & $3(1.45 \%)$ & $1(0.48 \%)$ \\
HMPV & $3(1.45 \%)$ & $1(0.48 \%)$ & $1(0.48 \%)$ & $1(3.38 \%)$ \\
MC & $3(1.45 \%)$ & $8(3.86 \%)$ & $4(1.93 \%)$ & $12(5.80 \%)$ \\
MP & $3(1.45 \%)$ & $4(1.93 \%)$ & $1(0.48 \%)$ & $2(0.97 \%)$ \\
PIV-1 & $1(0.48 \%)$ & 0 & $2(0.97 \%)$ & $3(1.45 \%)$ \\
PIV-2 & 0 & $1(0.48 \%)$ & 0 & $2(0.97 \%)$ \\
PIV-3 & $1(0.48 \%)$ & $1(0.48 \%)$ & $1(0.48 \%)$ & $3(1.45 \%)$ \\
PIV-4 & $2(0.97 \%)$ & 0 & 0 & $2(0.97 \%)$ \\
RSV & $1(0.48 \%)$ & $1(0.48 \%)$ & $2(0.97 \%)$ & $20(9.66 \%)$ \\
SP & $9(4.35 \%)$ & $9(4.35 \%)$ & 0 & $1(0.48 \%)$ \\
GAS & $1(0.48 \%)$ & 0 & & \\
\hline
\end{tabular}


detected at a sensitivity of 100 copies/ $\mu$ l. Thus, the RTPCR MassARRAY method established in this study had high detection sensitivity. In the analysis of 207 clinical samples for the detection of respiratory tract pathogens, the positive detection rate was $27.54 \%$ (57/207). S. pneumoniae, $H$. influenzae, M. pneumoniae, and $M$. catarrhalis are the main pathogens of community-acquired pneumonia and acute bacterial infections [25], and the detection rates of these four pathogens were found to be higher than those of other bacteria, such as Bordetella pertussis and Legionella pneumoniae, in this study. It was also found that among the 20 cases of S. pneumoniae infection, there were only $9(45.0 \%)$ cases of single pathogen infection, and among 11 cases of dual and multiple infections, there were five cases of $M$. catarrhalis co-infection and four of $H$. influenzae co-infection, which indicated that co-infections between bacteria were relatively common. Furthermore, the antibiotic resistance of these three bacteria is different [26]. Comprehensive and detailed pathogen diagnosis is of great significance in guiding the rational use of antibiotics to avoid the inappropriate prescription of antibiotics. There were $21 / 207(10.1 \%)$ samples that tested positive for viruses (note: there were two samples co-infected by three viruses). In China, viral infections are highly prevalent in autumn and winter and relatively low in spring and summer [27]. The samples in this study were collected from clinical patients from April to July (during spring and summer), which may be one of the reasons for the low positive rate of viral respiratory tract infections. PIV was detected in ten cases, including two cases of PIV1, three of PIV2, two of PIV3, and three of PIV4. There was no significant difference in the number of subtypes, which was different from the positive detection rates of PIV types 1, 2, 3, and 4 reported by Wang et al. (2019) in Shanghai, of 2.74, 0.62, 8.59, and $3.40 \%$, respectively [28]. This may be because that Wang et al.'s study population was mainly children, and PIV3 is one of the leading causes of lower respiratory tract infections in infants as well as immunocompromised people [29]. In this study, seven cases (3.38\%) of HMPV were detected, which was close to the positive detection rate of HMPV of $3.53 \%$ reported in 2018 by Zhong et al. (2019) [30]. We also detected six $(2.90 \%)$ cases of influenza A virus, all of which were H1N1. Additionally, respiratory syncytial virus was detected in two cases and adenovirus and human coronavirus in one case each. Due to the limited number of samples and sample types, some subtypes of human bocavirus; human enterovirus; influenza $\mathrm{B}$; and influenzae A H3, H3N2 seasonal, H5, and H7 were not detected in this study.

The MRT-MassARRAY respiratory pathogen detection system established in this study detected 27 respiratory pathogens and showed sensitivity and specificity similar to the results obtained using consensus PCR/RT-PCR. The advantage over real-time PCR is mainly reflected in the multiplicity of nucleic acid mass spectrometry amplification. In this study, a 27-plex one tube amplification mode was used, and the test cost was as low as 15 dollars per sample. MassARRAY detection uses 96/384 chips, with a high throughput of up to 384 samples. Based on this feature, our detection system is highly suited to early clinical pathogen screening and regional respiratory pathogen epidemiological investigations. A potential concern of this detection system is that a SNP could occur at the extension position which will lead to a nonspecific peak arising in the mass window, with its molecular weight not being consistent with the molecular weight in Table 2. Actually, this is a very rare situation that we had not encountered in this study, in case it happens Sanger sequencing of the PCR product would have to be done to determine the exact pathogen.

\section{Conclusion}

In summary, the analytical and clinical performance of the developed system based on MassARRAY technology for detecting 27 common respiratory pathogens is reliable and showing superior sensitivity, specificity, high throughput and user-friendly handling compared with other methods. It is a useful and cost-effective method, which have a good potential to be implemented for screening of respiratory pathogens and is of great significance in the clinical diagnosis of respiratory disease. Finally, large-scale population verification for the performance of MRT-PCRMassARRAY will facilitate its clinical application.

\section{Acknowledgements \\ We would like to thank Enago (http://www.enago.jp) for the English language review.}

\section{Statement \\ All methods in this study were carried out in accordance with relevant guidelines and regulations.}

\section{Authors' contributions}

Conceptualization: WC, XYR. Data curation: YCY, HZ. Formal analysis: YCY, HZ. Investigation: HZ, JFL. Methodology: YCY, JFL. Project administration: WC, XYR. Resources: HZ, JFL. Software: YCY. Supervision: XYR. Validation: HZ, JFL. Writing - original draft: HZ. Writing - review \& editing: WC, XYR. The authors read and approved the final manuscript.

\section{Funding}

No funds were received for the realisation of this work.

\section{Availability of data and materials}

The data that support the findings of this study are available from the corresponding author, upon request.

\section{Declarations}

Ethics approval and consent to participate

The collection of all NPA samples was approved by the ethics committee of the sixth people's hospital of Nantong. An informed consent was obtained from all subjects including minors (participants aged less than 18) who was obtained from parents or legal guardians. 


\section{Consent for publication}

Not applicable.

\section{Competing interests}

The authors declare that they have no conflicts of interest.

\section{Author details}

'Department of Respiratory Medicine, The Sixth People's Hospital of Nantong, Nantong, Jiangsu, China. ${ }^{2}$ Research and Development Centre, Hangzhou DiAn Medical Laboratory, Hangzhou, Zhejiang, China.

${ }^{3}$ Department of Respiratory Diseases, Nantong Tongzhou People's Hospital, Nantong, Jiangsu, China.

Received: 4 February 2021 Accepted: 18 July 2021

Published online: 25 August 2021

\section{References}

1. GBD. Lower Respiratory Infections Collaborators. Estimates of the global, regional, and national morbidity, mortality, and aetiologies of lower respiratory infections in 195 countries, 1990-2016: a systematic analysis for the Global Burden of Disease Study 2016. Lancet Infect Dis. 2018;18(11): 1191-210. https://doi.org/10.1016/S1473-3099(18)30310-4.

2. World Health Organization. Global health estimates 2016: deaths by cause, age, sex, by country and by region, 2000-2016. Geneva: World Health Organization; 2018.

3. Liapikou A, Torres A. Emerging drugs for nosocomial pneumonia. Expert Opin Emerg Drugs. 2016;21(3):331-41. https://doi.org/10.1080/14728214.201 6.1206077.

4. Boncristiani HF, Criado MF, Arruda E. Respiratory viruses. Encycl Microbiol. 2009;500-18. https://doi.org/10.1016/B978-012373944-5.00314-X.

5. Hendaus MA, Jomha FA, Alhammadi AH. Virus-induced secondary bacterial infection: a concise review. Ther Clin Risk Manag. 2015;11:1265-71. https:// doi.org/10.2147/TCRM.S87789.

6. Buchan BW, Ledeboer NA. Emerging technologies for the clinical microbiology laboratory. Clin Microbiol Rev. 2014;27(4):783-822. https://doi. org/10.1128/CMR.00003-14.

7. Zhang H, Morrison S, Tang YW. Multiplex polymerase chain reaction tests for detection of pathogens associated with gastroenteritis. Clin Lab Med. 2015;35(2):461-86. https://doi.org/10.1016/j.cll.2015.02.006.

8. Salez N, Vabret A, Leruez-Ville M, Andreoletti L, Carrat F, Renois F, et al. Evaluation of four commercial multiplex molecular tests for the diagnosis of acute respiratory infections. PLoS One. 2015;10(6):e0130378. https://doi.org/1 0.1371/journal.pone.0130378.

9. Poritz MA, Blaschke AJ, Byington CL, Meyers L, Nilsson $K$, Jones $D$, et al. FilmArray, an automated nested multiplex PCR system for multipathogen detection: development and application to respiratory tract infection. PLoS One. 2011;6(10):e26047. https://doi.org/10.1371/journal. pone.0026047.

10. Chen JHK, Lam HY, Yip CCY, Wong SCY, Chan JFW, Ma ESK, et al. Clinical evaluation of the new high-throughput Luminex NxTAG respiratory pathogen panel assay for multiplex respiratory pathogen detection. J Clin Microbiol. 2016;54(7):1820-5. https://doi.org/10.1128/JCM.00517-16.

11. Millis MP. Medium-throughput SNP genotyping using mass spectrometry: multiplex SNP genotyping using the iPLEX gold assay. Methods Mol Bio. 2011;700:61-76. https://doi.org/10.1007/978-1-61737-954-3_5.

12. Svidnicki MC, Silva-Costa SM, Ramos PZ, dos Santos NZP, Martins FTA, Castilho AM, et al. Screening of genetic alterations related to nonsyndromic hearing loss using MassARRAY iPLEX technology. BMC Med Genet. 2015;16(1):85. https://doi.org/10.1186/s12881-015-0232-8.

13. Chen HY, Liu CH, Chang YH, Yu SL, Ho BC, Hsu CP, et al. EGFR-activating mutations, DNA copy number abundance of ErbB family, and prognosis in lung adenocarcinoma. Oncotarget. 2016;7(8):9017-25. https://doi.org/10.1 8632/oncotarget.7029.

14. Yin L, Cai WJ, Liu CX, Chen YZ, Hu JM, Jiang JF, et al. Analysis of PTEN methylation patterns in soft tissue sarcomas by MassARRAY spectrometry. PLOS ONE. 2013;8(5):e62971.

15. Weber S, Spiegl B, Perakis SO, Ulz CM, Abuja PM. Technical evaluation of commercial mutation analysis platforms and reference materials for liquid biopsy profiling. Cancers (Basel). 2020;12(6):1588.

16. Coiras MT, Aguilar JC, García ML, Casas I, Pérez-Breña P. Simultaneous detection of fourteen respiratory viruses in clinical specimens by two multiplex reverse transcription nested-PCR assays. J Med Virol. 2004;72(3): 484-95. https://doi.org/10.1002/jmv.20008.

17. Pretorius MA, Madhi SA, Cohen C, Naidoo D, Groome M. Respiratory viral coinfections identified by a 10-plex real-time reverse-transcription polymerase chain reaction assay in patients hospitalized with severe acute respiratory illness-South Africa, 2009-2010. J Infect Dis. 2012;206(Suppl 1): S159-65. https://doi.org/10.1093/infdis/jis538.

18. Bellau-Pujol S, Vabret A, Legrand L, Dina J, Gouarin S. Development of three multiplex RT-PCR assays for the detection of 12 respiratory RNA viruses. J Virol Methods. 2005;126(1-2):53-63. https://doi.org/10.1016/j.jviromet.2005. 01.020 .

19. Echavarria M, Forman M, Ticehurst J, Dumler JS, Charache P. PCR method for detection of adenovirus in urine of healthy and human immunodeficiency virus-infected individuals. J Clin Microbiol. 1998;36(11): 3323-6. https://doi.org/10.1128/JCM.36.11.3323-3326.1998.

20. Kapoor A, Simmonds P, Slikas E, Li L, Bodhidatta L, Sethabutr O, et al. Human bocaviruses are highly diverse, dispersed, recombination prone, and prevalent in enteric infections. J Infect Dis. 2010;201(11):1633-43. https://doi. org/10.1086/652416.

21. Vabret A, Dina J, Gouarin S, Petitjean J, Corbet S, Freymuth F. Detection of the new human coronavirus HKU1: a report of 6 cases. Clin Infect Dis. 2006; 42(5):634-9. https://doi.org/10.1086/500136.

22. Van der Hoek L, Pyrc K, Jebbink MF, Vermeulen-Oost W, Berkhout RJ, et al. Identification of a new human coronavirus. Nat Med. 2004;10(4):368-73. https://doi.org/10.1038/nm1024.

23. Viazov S, Ratjen F, Scheidhauer R, Fiedler M, Roggendorf M. High prevalence of human Metapneumovirus infection in young children and genetic heterogeneity of the viral isolates. J Clin Microbiol. 2003;41(7):3043-5. https://doi.org/10.1128/JCM.41.7.3043-3045.2003.

24. Zhu Y, Zhu X, Deng M, Wei H, Zhang M. Causes of death in hospitalized children younger than 12 years of age in a Chinese hospital: a 10 year study. BMC Pediatr. 2018;18(1):8. https://doi.org/10.1186/s12887-017-0981-y.

25. Dunne EM, Manning J, Russell FM, Robins-Browne RM, Mulholland EK. Effect of pneumococcal vaccination on nasopharyngeal carriage of Streptococcus pneumoniae, Haemophilus influenzae, Moraxella catarrhalis, and Staphylococcus aureus in Fijian children. J Clin Microbiol. 2012;50(3):1034-8. https://doi.org/10.1128/JCM.06589-11.

26. Perera W, Ling C, Mchugh T, Donaldson GC, Wedzicha JA. Quantitative detection of $\mathrm{S}$ pneumoniae, $\mathrm{H}$ influenzae, and $\mathrm{M}$ catarrhalis in sputum samples from chronic obstructive pulmonary disease patients by real-time polymerase chain reaction, Winter Meeting of the British Thoracic Society/ British Lung Foundation BMJ Publishing; 2006. p. 1128.

27. Chan PKS, Tam WWS, Lee TC, Hon KL, Lee N. Hospitalization incidence, mortality and seasonality of common respiratory viruses over a period of 15 years in a developed subtropical city. Med (Baltim). 2015;94(46):e2024. https://doi.org/10.1097/MD.0000000000002024.

28. Chun W, Fen P, Yingying S. Epidemic charactristics and clinical symptoms of parainfluenza virus in hospitalized children with acute lower respiratory tract infection. Int J Virol. 2019;8(26):26-31.

29. Xin Q, Xinfen Y, Min Z. Genetic evolution and infection characteristics of parainfluenza virus type 3 in infants with acute respiratory infection in Hangzhou. Chin J Prev Med. 2016;50(3):255-60.

30. Jiayu Z, Weiying C, Jiahui X. Epidemiological analysis of Metapneumovirus, bocavirus and adenovirus in acute respiratory infection in Guangzhou from 2016 to 2018. Chin Biomed Eng. 2019;25(2):237-9.

\section{Publisher's Note}

Springer Nature remains neutral with regard to jurisdictional claims in published maps and institutional affiliations. 